\title{
Prediction of Leakage from an Axial Piston Pump Slipper with Circular Dimples Using Deep Neural Networks
}

\author{
Özkan Özmen ${ }^{1 *}$ (D), Cem Sinanoğlu', Abdullah Caliskan² and Hasan Badem³
}

\begin{abstract}
Oil leakage between the slipper and swash plate of an axial piston pump has a significant effect on the efficiency of the pump. Therefore, it is extremely important that any leakage can be predicted. This study investigates the leakage, oil film thickness, and pocket pressure values of a slipper with circular dimples under different working conditions. The results reveal that flat slippers suffer less leakage than those with textured surfaces. Also, a deep learning-based framework is proposed for modeling the slipper behavior. This framework is a long short-term memory-based deep neural network, which has been extremely successful in predicting time series. The model is compared with four conventional machine learning methods. In addition, statistical analyses and comparisons confirm the superiority of the proposed model.
\end{abstract}

Keywords: Slipper, Leakage, Circular dimpled, Long short-term memory, Deep neural network

\section{Introduction}

Axial piston pumps are frequently used in hydraulic applications which require high pressures. In the axial piston pump shown in Figure 1, one of the most important locations for metal-metal contact is between the slipper and the swash plate.

This friction couple is lubricated hydrodynamically/ hydrostatically depending on the piston geometry and the slipper pocket ratio, and the forces on the piston are balanced on this surface. The texture of the surface, however, directly affects the performance of the tribological surfaces which function through lubrication. Nevertheless, obtaining the optimum surface texture design depends heavily on the geometry and the working conditions of the surfaces. Additionally, oil can be retained on these surfaces, and the resulting contamination can be maintained in the pits on the surface, thus extending the life of the mechanical parts [1].

\footnotetext{
*Correspondence: ozmen@erciyes.edu.tr

${ }^{1}$ Tribology Laboratory, Industrial Design Engineering, Faculty

of Engineering, Erciyes University, Kayseri 38039, Turkey

Full list of author information is available at the end of the article
}

A multitude of studies on the surface of hydrodynamic and hydrostatic bearings have been conducted over the past 30 years, including analytical, experimental, numeri$\mathrm{cal}$, and artificial intelligence investigations. For example, Koç et al. [2, 3] proposed that the slipper should have a small convex surface to enable successful performance, whereas Bergada et al. [4, 5] conducted an analytical study to understand the lifting property and leakage of a slipper containing a number of grooves. These grooves on the slipper change the pressure distribution, leakage, and lifting force acting on the slipper. Etsion et al. [6] considered a textured surface for the parallel thrust bearing, and observed that the friction coefficient was lower in partially textured bearings. Sharma et al. [7] performed numerical simulations for fully and partially textured thrust pad bearings with different lubricants, and reported that micro-surface textures cause slight changes in load-bearing capacity, but made a significant difference in frictional power dissipation. Ye et al. [8] numerically examined the load-carrying capacity under different operating conditions to investigate the effect of elasto-hydrodynamic lubrication caused by the pressure distribution of a textured slipper. They observed that 


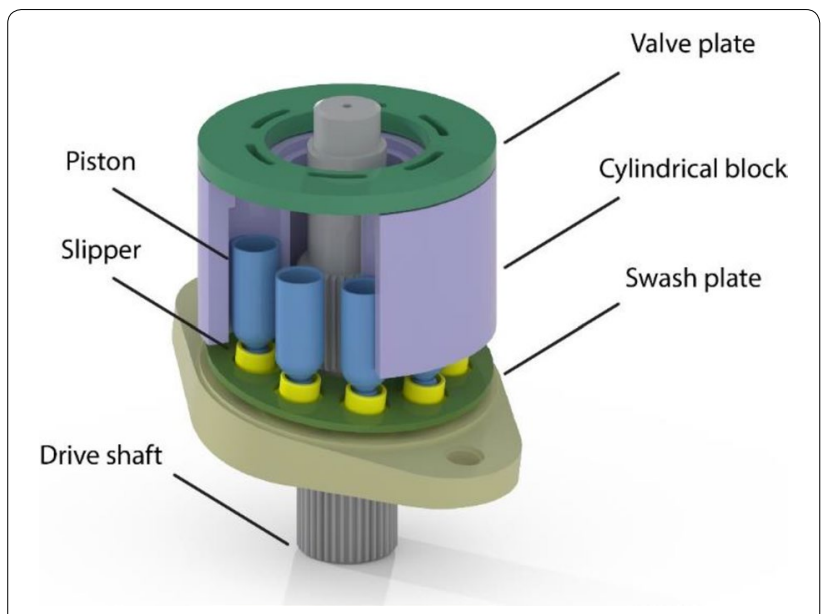

Figure 1 Axial piston pump configuration

the maximum stiffness coefficient and optimum oil film thickness could be determined by operating parameters. Also, as loading pressure and rotational speed increases, the collective dimple effect of a textured slipper increase and creates a thicker oil film thickness. As a result, it would result in a higher leakage rate.

Although analytical and numerical solutions enhance our understanding of the physical phenomena occurring between the slipper and swash plate, they are based on certain assumptions. When the inertial effects of solids and fluids, thermal factors, and vibration are considered, mathematical solutions become more difficult. In such cases, machine learning methods (MLMs) offer the ability to model non-linear systems without any assumptions. The use of a neural network (NN) for axial piston pumps was first realized by Kakaub et al. [9]. In their study, the data obtained from an experimental setup was used to reduce the power loss under high pressures. They demonstrated that an $\mathrm{NN}$ trained using Levenberg-Marquardt optimization was able to predict the steady-state and dynamic behavior of the pump accurately. In another study, an $\mathrm{NN}$ was employed to predict the pressure distribution and load-bearing capacity [10]. The results demonstrated that NNs are more effective than theoretical models for determining the pressure distribution and load-bearing capacity. Canbulut et al. [11] reported an experimental study of the load bearing capacity, leakage, friction moment, and pump power properties of the slipper under different working conditions, and developed an NN to predict the results. This NN exhibited superior performance at predicting the static and dynamic parameters of the bearing system. In another study by Canbulut et al. [12], the effects of the surface roughness of the slippers on lubrication were studied experimentally. They also observed that surface roughness has important effects on leakage. In addition, they designed the behavior of this slipper using an $\mathrm{NN}$, and argued that their model could be used in real-time applications. Another study investigated the power dissipation of conical and flat-surfaced slippers and observed that the slippers with flat surfaces performed better. Moreover, the power dissipation was modeled using an NN, which was found to adapt very well to experimental data [13]. Canbulut et al. [14] also studied the frictional power loss of the slipper theoretically and experimentally. They observed that the study parameters and surface geometry contribute to the frictional power loss, and formed an NN model for frictional power loss with high predictive capabilities. Ozmen et al. [15] modeled the pressure distribution and leakage of a flat slipper working hydrostatic/hydrodynamically using Multi-Gene Genetic Programming (MGGP) and NN, MLMs. The results showed that both MLMs had high predictive performance in terms of pressure distributions and leakage for a flat slipper. However, analytical equations showed lower performance than MLMs because of neglected of flow inertia and system noise. Following an experimental analysis of the hydrodynamic and general efficiency variations of gear pumps, vane pumps, and axial pumps, an NN prediction model was developed to act as a predictor in the applications of such systems [16].

The objective of this study is to experimentally research the leakage, oil film thickness, and pocket pressure of different slipper surface geometries under various working conditions. Although many NNs have been used to predict the complex relationship between the slipper and swash plate, they typically require a high number of external parameters to be entered, their performance is directly dependent on the optimization method, and they are extremely susceptible to noisy data. To overcome these disadvantages, we investigate the application of a long short-term memory (LSTM)-based deep neural network (DNN), which offers superior performance in predicting time series. Compared with conventional MLMs, DNNs can achieve superior classification and modeling performance [17]. To the best of our knowledge, DNNs have not yet been used for this purpose. The results derived in this study have been compared with those from conventional MLMs such as linear regression (LR), decision tree (DT), support vector machine (SVM), and NN. The proposed model is statistically proven to be superior to all of these methods.

This article is structured as follows. In Section 2, the working principle, experimental setup, and experimental method of axial piston pumps are briefly summarized. Next, in Section 3, the DNN prediction model is 
explained. Section 4 presents and discusses the experimental results, and Section 5 gives our conclusions and ideas for future work.

\section{Theorical Background and Experimental Setup}

In the axial piston pump shown in Figure 1, when the cylindrical block turns, the piston moves back and forth through the swash plate, enabling oil to be pumped under high pressures. Some of the oil is used to balance the forces on the piston and to prevent metal-metal contact between the slipper and swash plate by means of the ball joint and the orifice. This friction couple directly impacts the pump performance [18]. Assuming that the bearing pocket is sufficiently large for the oil film thickness on slipper land, the pressure distribution for flat slippers can be theoretically derived from the Reynolds lubrication equation in cylindrical coordinates:

$$
P=P_{r} \frac{\ln \left(\frac{r}{r_{o}}\right)}{\ln \left(\frac{r_{i}}{r_{o}}\right)},
$$

when the slipper pocket pressure, geometric dimensions, dynamic viscosity of oil, and fixed oil thickness are considered, the theoretical leakage can be derived as [11]:

$$
Q=\frac{P_{r} \cdot \pi \cdot h^{3}}{6 \cdot \eta \cdot \ln \left(\frac{r_{o}}{r_{i}}\right)} .
$$

A multitude of experimental setups has been prepared to study the different parameters between the slipper and swash plate, as they have a direct effect on pump performance [18]. In the present study, the experimental setup in Figure 2a is used to investigate the slipper leakage, pocket pressure, and oil film thickness under various working conditions.

Figure $2 \mathrm{~b}$ shows the disassembly of the main test unit. The hydraulic loading cylinder is designed in such a way as to load three slippers equally. The swash plate, made of steel, has an axial bearing and its surface has been sanded. The swash plate is driven by a $5 \mathrm{~kW}$ servo motor with a working range of $0-2300 \mathrm{r} / \mathrm{min}$. The swash plate has a mean surface roughness of $1.33 \mu \mathrm{m}$. The slipper in Figure 3 is made of brass, and its dimensions are listed in Table 1.

The average oil film thickness and average runout were measured on one of the slippers using Mitutoyo's Absolute Digimatic Indicator with Output Comparator, which has a sensitivity of $1 \mu \mathrm{m}$ (Figure 3). On the other slipper, the pressures were measured by KellerPA-21-SR 0-6 $\mathrm{MPa}$ piezoresistive pressure sensors placed on the measuring holes, with one in the slipper pocket and the others located $1.5 \mathrm{~mm}$ inward from the outer radius at an angle

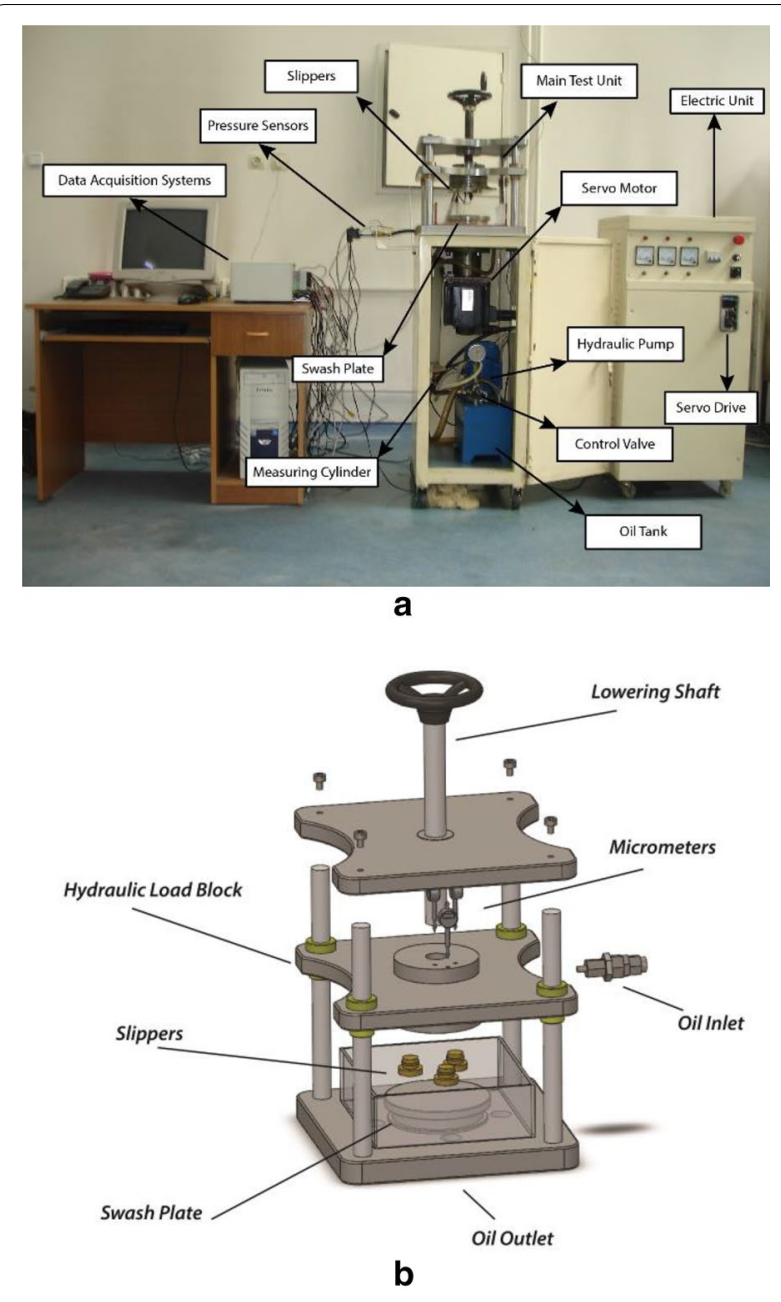

Figure $\mathbf{2}$ a Experimental setup and $\mathbf{b}$ schematic disassembly of the main test unit

of $120^{\circ}$ and a hole diameter of $0.6 \mathrm{~mm}$. In addition, the supply pressure was measured with a pressure sensor. The temperatures of the oil on the slipper and in the tank were measured with a thermocouple. To measure the leakage, a flow meter was mounted on the oil inlet section, and the same leakage values were recorded with a measuring cylinder. A fixture was prepared for the slipper, and circular dimples were formed on the surface of the slipper using a face-milling machining production method. In these experiments, the surface of the same slipper was milled and used again. Thus, any differences resulting from manufacturing have been eliminated. Data from the experimental setup were collected a 20-channel data logger adjusted to give one datum per second (AHLBORN, Figure 2a).

For the experiments, circular dimples (Type 1: $1 \mathrm{~mm}$ in diameter and depth; Type 2: $1 \mathrm{~mm}$ in diameter and 


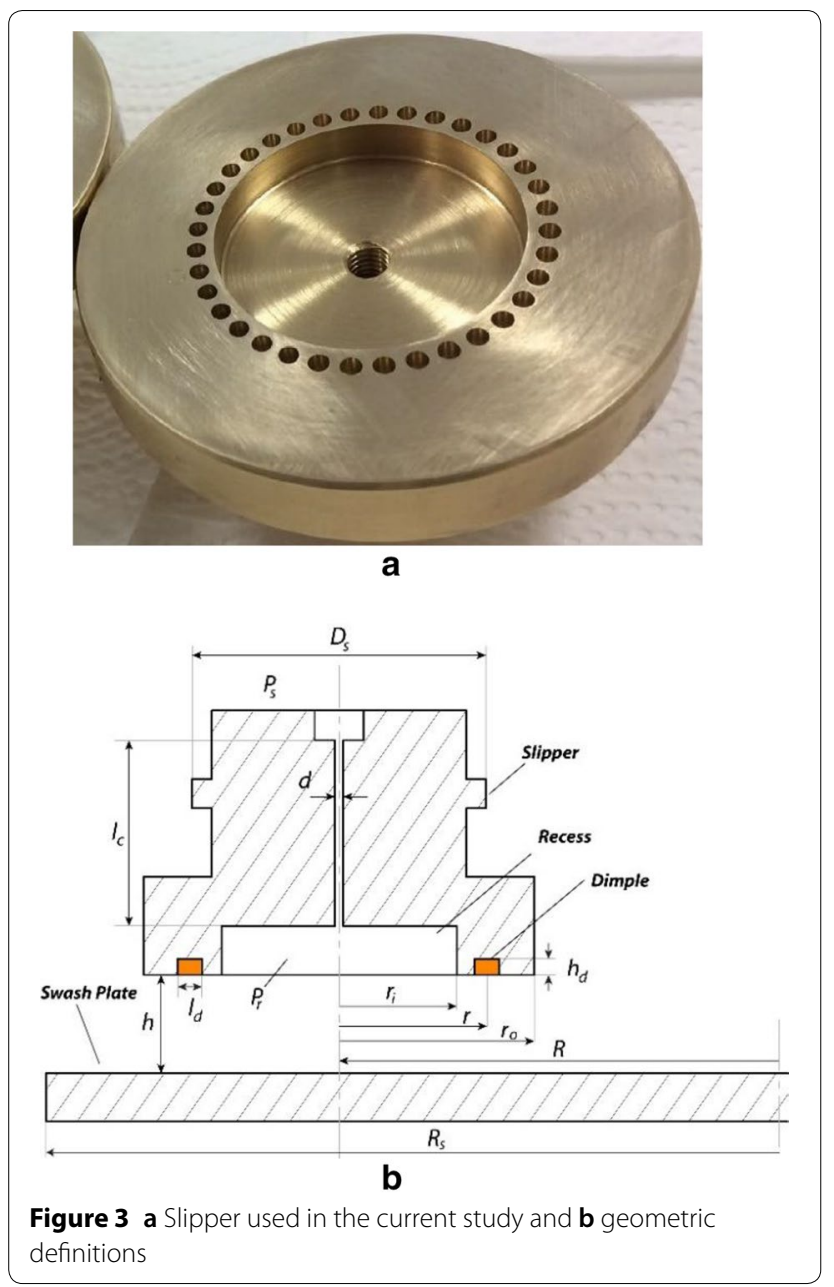

Table 1 Dimensions of slipper and swash plate used in experiments

\begin{tabular}{lll}
\hline Symbol & Parameter & Value \\
\hline$r_{i}$ & Inner radius & 15 \\
$r_{0}$ & Outer radius & 30 \\
$R$ & Rotation radius & 45 \\
$R_{s}$ & Swash plate radius & 86 \\
$d$ & Diameter of the capillary tube & 0.6 \\
$l_{C}$ & Length of the capillary tube & 20 \\
$h_{d}$ & Length of the circular dimple & $0-1-2^{*}$ \\
$l_{d}$ & Diameter of the circular dimple & 1 \\
$r$ & Radius of the circular dimple center & 16.5 \\
$D_{s}$ & Piston diameter & 30 \\
$\alpha$ & Angle between two circular dimple center & 10 \\
\hline
\end{tabular}

Units of lengths are $\mathrm{mm}$

Units of angle are degrees

*0 shows flat slipper
$2 \mathrm{~mm}$ in depth) were engraved in the slipper and the results were compared with those from a flat slipper. The circular dimples were formed close to the slipper pocket, so that their effect could be better observed [19]. In the experiments, the temperature of the Shell Tellus 68 hydraulic fluid was kept constant at $25{ }^{\circ} \mathrm{C}$ $\left(\eta=0,12278 \mathrm{~N} \cdot \mathrm{s} / \mathrm{m}^{2}\right)$ by means of an air-cooled system. In addition, each experiment was repeated three times. The free up and down movement of the slipper when pressure oil is administered to the system arranges oil film thickness. Experiments were performed under supply pressures of 4,5 , and $6 \mathrm{MPa}$ and at turning speeds of $250,500,750,1000$, and $1250 \mathrm{r} / \mathrm{min}$. The mean of these data (45 data) were used for experimental comparisons, and the full 7215 data points recorded by the data logger were used to form the prediction models, thus satisfying the high dimensionality required by DNNs [17]. Note that the second row of circular dimples made in the slippers lifted the slippers excessively, so that the supply pressure could not exceed $5.5 \mathrm{MPa}$. For this reason, the location and dimensions of the circular dimples in the slipper have been restricted with the parameters mentioned above.

\section{Deep Neural Network Model}

Deep learning is very useful in many machine learning tasks, including speech, image, and signal analysis as well as numerous classification problems [17, 20-23]. There are a number of DNN architectures, including convolutional neural networks, stacked autoencoders, and Boltzmann machines. However, there have been limited attempts to use DNNs for regression problems. In this paper, we present a framework for the prediction of leakage, pocket pressure, and oil film thickness from the experimental values obtained from the experimental setup described in Section 2.

The analytical equations for the interaction between the slippers/swashplate are obtained under the specific assumptions of the Reynolds lubrication equations in cylindrical coordinates. However, in reality, solid and flow inertia, vibration, noise, and friction between parts all have an effect on the flow. For this reason, we propose a new technique for the prediction of these parameter values using MLMs instead of analytical approaches. The proposed framework is based on LSTM, which is an improved version of the recurrent neural network (RNN).

\subsection{Recurrent Neural Network}

RNNs are DNNs consisting of several basic layers. Both past and current information are utilized by a RNN [24] during the training stage, unlike traditional Feed-Forward Neural Networks (FFNNs). RNNs have three layers, namely, input, hidden, and output layers. One of the most 
important issues encountered in the training of RNNs is the gradient vanishing problem, in which the gradient of the network becomes close to zero during the back-propagation algorithm.

\subsection{LSTM Neural Network}

LSTM is an improved RNN that overcomes the gradient vanishing problem suffered by RNNs [25]. However, this improvement increases the complexity of the network. Although the structure of LSTM is similar to that of RNNs, new control parameters including input, output, and forget gates are added to the LSTM network to control the flow of information between the input time series. The cell structure of the LSTM is shown in Figure 4 , where $\mathbf{x}^{\langle\mathrm{T}\rangle}$ is the D-dimensional input vector, $\mathbf{h}^{\langle\mathrm{T}\rangle}$ is the output of the LSTM cell, and $\mathbf{c}^{\langle\mathrm{T}\rangle}$ defines the cell state.

The LSTM cell can take one of two cell states. The first is the hidden state, also known as the output state. The second is the cell state denoted by c. In the hidden state, the output value of the network is obtained from the input series using the cell state, which controls the information flow from past series. There are four important parts inside the LSTM cell: the input gate (i), forget gate (f), cell candidate (g), and output gate (o). These gates are similar to those found in a regular FFNN $[25,26]$.

1. The input gate, which is used to update the cell state, is defined as follows:

$$
\mathbf{i}^{\langle\mathrm{T}\rangle}=f_{g}\left(\mathbf{W}_{i} \mathbf{x}^{\langle\mathrm{T}\rangle}+\mathbf{R}_{i} \mathbf{h}^{\langle\mathrm{T}-1\rangle}+\mathbf{b}_{i}\right),
$$

where $\mathbf{i}^{\langle T\rangle}$ is the output of the input gate, and $\mathbf{W}_{i}, \mathbf{R}_{i}, \mathbf{b}_{\boldsymbol{i}}$ are the input weights, recurrent weights, and the bias of the input gate, respectively.

2. The forget gate determines the level of the cell state reset, and is given as follows:

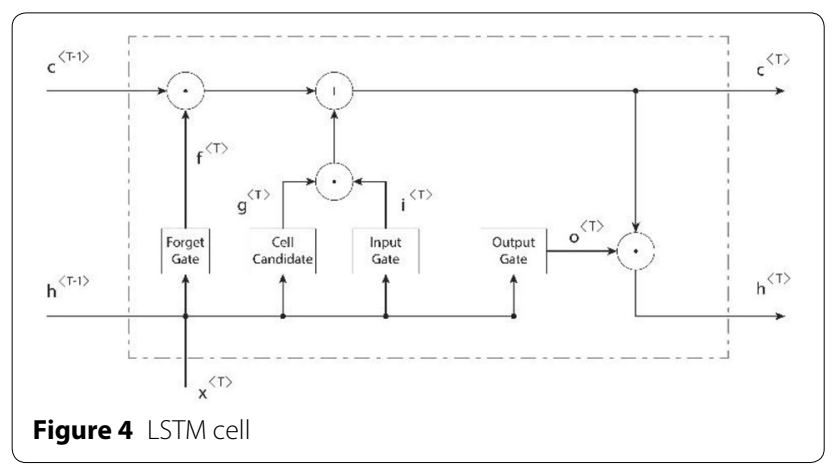

$$
\mathbf{f}^{\langle\mathrm{T}\rangle}=f_{g}\left(\mathbf{W}_{f} \mathbf{x}^{\langle\mathrm{T}\rangle}+\mathbf{R}_{f} \mathbf{h}^{\langle\mathrm{T}-1\rangle}+\mathbf{b}_{f}\right),
$$

where $\mathbf{f}^{\langle T\rangle}$ is the output of $\mathbf{W}_{f}, \mathbf{R}_{f}, \mathbf{b}_{f}$ the forget gate, and are the input weights, recurrent weights, and the bias of the forget gate, respectively.

3. The cell candidate adds information to the cell state, and is defined as follows:

$$
\mathbf{g}^{\langle\mathrm{T}\rangle}=f_{c}\left(\mathbf{W}_{g} \mathbf{x}^{\langle\mathrm{T}\rangle}+\mathbf{R}_{g} \mathbf{h}^{\langle\mathrm{T}-1\rangle}+\mathbf{b}_{g}\right),
$$

where $\mathbf{g}^{\langle\mathrm{T}\rangle}$ is the output of the cell candidate, and $\mathbf{W}_{g}, \mathbf{R}_{g}$, $\mathbf{b}_{g}$ are the input weights, recurrent weights, and bias of the cell candidate unit, respectively.

4. The output gate controls the amount of cell state information added to the hidden state:

$$
\mathbf{o}^{\langle\mathrm{T}\rangle}=f_{g}\left(\mathbf{W}_{o} \mathbf{x}^{\langle\mathrm{T}\rangle}+\mathbf{R}_{o} \mathbf{h}^{\langle\mathrm{T}-1\rangle}+\mathbf{b}_{o}\right),
$$

where $\mathbf{o}^{\langle\mathrm{T}\rangle}$ is the output of the output gate, and $\mathbf{W}_{o}, \mathbf{R}_{o}$, $\mathbf{b}_{o}$ are the input weights, recurrent weights, and bias of the output gate, respectively. $f_{g}$ and $f_{c}$ denote the sigmoid function and hyperbolic tangent function, respectively.

Along with the four equations given above, the following two equations are used to generate the output of the LSTM cell:

$$
\begin{aligned}
& \mathbf{c}^{\langle\mathrm{T}\rangle}=\mathbf{f}^{\langle\mathrm{T}\rangle} \odot \mathbf{c}^{\langle\mathrm{T}-1\rangle}+\mathbf{i}^{\langle\mathrm{T}\rangle} \odot \mathbf{g}^{\langle\mathrm{T}\rangle}, \\
& \mathbf{h}^{\langle\mathrm{T}\rangle}=\mathbf{o}^{\langle\mathrm{T}\rangle} \odot f_{c}\left(\mathbf{c}^{\langle\mathrm{T}\rangle}\right),
\end{aligned}
$$

where $\odot$ denotes element-wise multiplication.

\subsection{Proposed DNN Framework}

DNN has several user-supplied control parameters, including, batch size, number of iterations, hidden size, the number of layer, etc. However, there is no theoretical way to choose these parameters to be tuned heuristically [23]. As an example, we have observed the performance of the proposed network with respect to first and second hidden sizes. Figure 5 shows that the accuracy of the pocket pressure according to the different number of neurons in the first and second hidden layers. So the proposed framework has five layers: a normalization layer, two LSTM layers, a fully connected layer, and a regression layer. The network shown in Figure 6 is called the deep-LSTM network. The input to the network is 

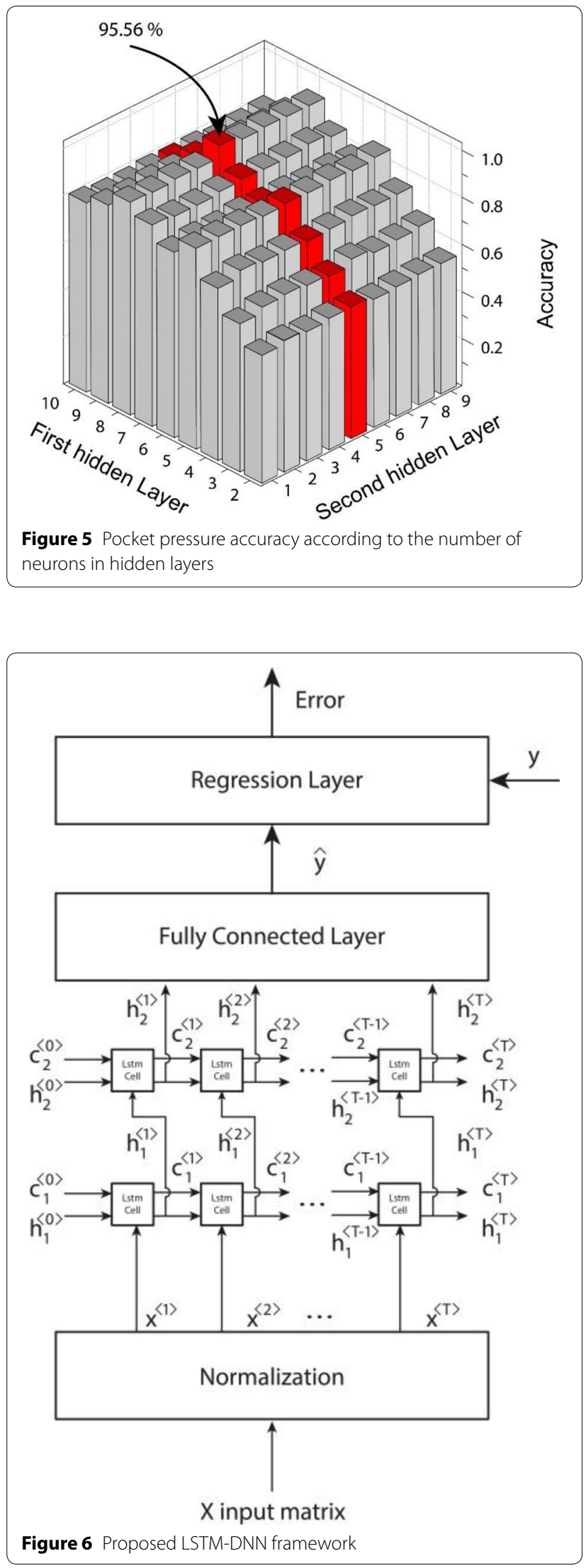

normalized to have zero mean and a standard deviation of 1 using the normalization layer. Thus, all features are set on the same scale. For simplicity, the LSTM layers can be thought of as repetitions of the cell described in the previous section [26]. The input to the first LSTM layer is the normalized time series of the input $X$, whose past and current information is employed in this layer to generate the first hidden layer vector $\boldsymbol{h}_{1}$. To generate the output of the second LSTM layer, $\boldsymbol{h}_{1}$ is fed into the second LSTM layer as an input time series.

The second LSTM layer generates $\boldsymbol{h}_{2}$ for input to the fully connected layer, which is a regular FFNN whose output is the predicted output $\hat{\boldsymbol{y}}$. The loss between the predicted value $\hat{y}$ and the real value $\boldsymbol{y}$ is calculated by the error function:

$$
e=\frac{1}{T} \sum_{i=1}^{T}\left(\hat{y}^{\langle T\rangle}-y^{\langle T\rangle}\right)^{2}
$$

where $T$ is the length of the time series and also the number of samples.

To determine the network weights, the back propagation algorithm is applied to the whole network. The main purpose is to decrease the error between the predicted and real values. The dataset in this study has been divided into two, with $70 \%$ used for training and $30 \%$ for testing. The parameters used for the DNN are summarized in Table 2.

To demonstrate the efficacy of the proposed method, simulations were repeated 30 times. This was done to confirm the effectiveness of the deep-LSTM statistically. As shown in Eqs. (10)-(12), the LR, DT, SVM, NN, and DNN models use the variable input data obtained from the data logger as the output data for the leakage, oil film thickness, and pocket pressure, respectively. The parameter values for the SVM, LR, NN, and DT models were those suggested in the literature. All the simulation processes were realized on computers equipped with an Intel

Table 2 Parameters of the proposed DNN

\begin{tabular}{llll}
\hline & Leakage & Oil film thic. & Pocket pres. \\
\hline Optimization method & sgdm* & sgdm* & sgdm* \\
Maximum iteration & 500 & 500 & 500 \\
Momentum & 0.9 & 0.9 & 0.9 \\
Initial learning rate & 0.02 & 0.04 & 0.05 \\
Learning rate drop period & 120 & 40 & 200 \\
Learning rate drop factor & 0.5 & 0.8 & 0.2 \\
Mini batch size & 128 & 128 & 128 \\
Hidden size first layer & 5 & 7 & 5 \\
Hidden size second layer & 8 & 5 & 8 \\
\hline
\end{tabular}

*Stochastic gradient descent method 
6700K 4.0 GHz CPU, 16 GB DDR4 RAM, and GTX 980 Ti 6 GB Nvidia GeForce DX12.

$$
\begin{aligned}
& Q_{r}=f\left(P_{s}, P_{r}, P_{1}, P_{2}, P_{3}, n, r_{r o_{1}}, r_{r o_{2}}, r_{r o_{3}}, h_{d}, l_{d}\right) \\
& h=f\left(P_{r}, Q_{r}, P_{1}, P_{2}, P_{3}, n, r_{r O_{1}}, r_{r O_{2}}, r_{r O_{3}}, h_{d}, l_{d}\right) \\
& P_{r}=f\left(P_{s}, Q_{r}, P_{1}, P_{2}, P_{3}, n, r_{r O_{1}}, r_{r o_{2}}, r_{r o_{3}}, h_{d}, l_{d}\right)
\end{aligned}
$$

\section{Results and Discussion}

As the flow rate between the slipper and swash plate is high, it is considered to be laminar. However, the effect on the leakage and the oil film thickness is proportional to the amplitude of the slipper/swash plate runout [4]. As explained in previous sections, owing to the oil pressure on the slipper, oil flows from the orifice towards the slipper pocket, and this oil results in pressure being applied in the slipper pocket. As the swash plate turns, the oil in the slipper flows over the slipper land and prevents metal-metal contact.

Figure 7 shows the leakage values obtained experimentally under different working conditions for the slipper with circular dimples. Experimental studies have generally shown that leakage increases as the supply pressure rises, and decreases slightly as the turning speed increases $[4,5]$. With the 1-mm-deep dimples on the slipper, all turning speeds and pressures resulted in an increase in the leakage. This is an undesirable occurrence in terms of pump efficiency. In the case of the 2-mmdeep circular dimples, however, the leakage followed a similar trend to that for flat slippers. As a result of the experimental study, leakage according to the depth/diameter ratio of the surface texture appeared similar to the theoretical work [8]. It has been observed that the leakage

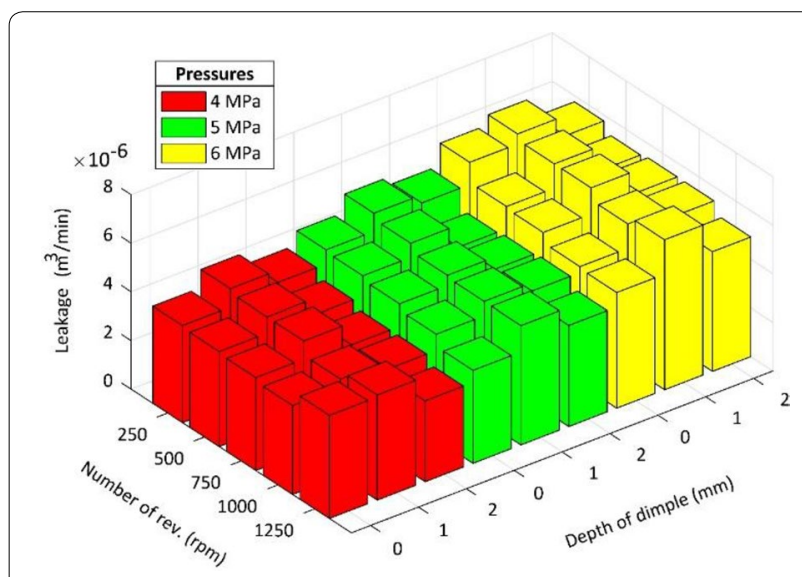

Figure 7 Leakage under different study parameters for different dimple depths increased with surface texture depth. However, the leakage decreases after a certain depth of surface texture.

This phenomenon has been explained in the literature through the vorticity created in slippers with grooves, and it has been reported that, for a certain groove width and groove position, the leakage increases in parallel with increases in the groove depth, but then remains constant above a certain groove depth [19]. In addition, these results show that the flow between the slippers/swashplate can be controlled by the surface texture.

Figure 8 shows the average oil film thickness. The oil film thickness is independent of the supply pressure, and decreases slightly as the turning speed increases. Theoretically, under adequate lubrication conditions, while the slipper is working, the oil film forms through the balance between the downward forces on the piston and the upward forces under the slipper. In a slipper with a depth of $1 \mathrm{~mm}$, where the leakage is as high in Figure 7, the oil film thicknesses are also high under all working conditions. The increase in oil film thickness, however, will diminish the friction power loss [27].

Figure 9 shows the pocket pressure of the slipper. Although the pocket pressure of the slipper does not change much compared to the turning speed, it does increase in parallel with the increase in supply pressure. However, the increase in the oil film thickness between the slipper and the swash plate acts to lower the pocket pressure. The lowest pocket pressure for the cases studied was measured in the slipper with a dimple surface depth of $1 \mathrm{~mm}$. This is to be expected, as the leakage and oil film thickness are high in this case.

The experimental studies described above were also modeled by means of the MLMs described in Section 3. The models were executed 30 times independently, and the mean, maximum, minimum, and standard deviation

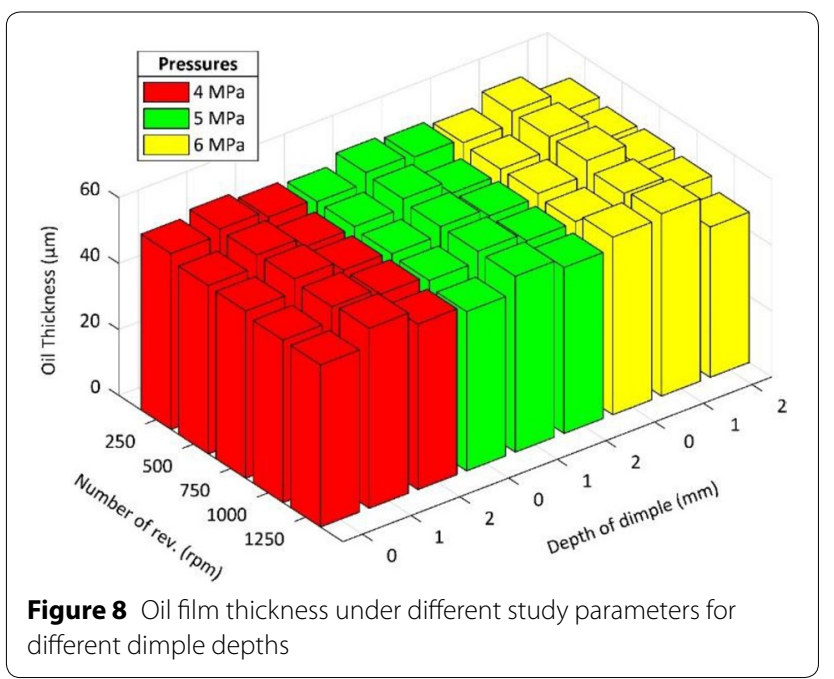




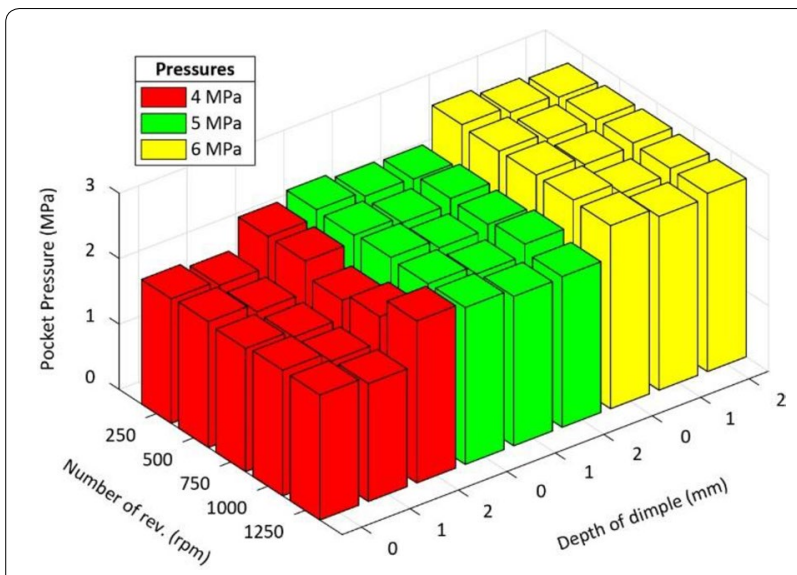

Figure 9 Pocket pressure values under different study parameters for different dimple depths

values are presented in Tables 3 and 4. A comparison of the results and the conformity of the DNN model to 100 test data are presented in Figure 10. Figure 10a shows the root mean square error (RMSE) in the leakage obtained from running the models. As seen in the figure and tables, the DNN model achieved lower RMSE values than LR, DT, SVM, and NN models. Figure 10a also shows that the DT and NN models give similar predictions, and that the LR model yields the highest RMSE and the lowest $\mathrm{R}^{2}$ value in all trials. Although all MLMs generally gave high mean $R^{2}$ values, the suggested LSTM-based DNN yields the best result with the lowest RMSE. Figure 10b shows the RMSE values for the oil film thickness. As shown in the figure and tables, the $\mathrm{NN}$ and DNN models yield lower RMSE and higher $R^{2}$ values than the other three models in all cases, whereas DT gives the highest RMSE value. However, with its highest $\mathrm{R}^{2}$ and lowest RMSE values, the DNN model is clearly able to generate better predictions than the other models. Figure 10c shows the RMSE values for the pocket pressure. The RMSE values are close to one another in this case, although the LR model has the highest error value and the lowest $R^{2}$ value. This study of the mean values has revealed that, although the $\mathrm{R}^{2}$ values of all the models are quite high, the DNN model produces the lowest RMSE value for each of the three slipper parameters, and thus makes the best predictions.

Although the suggested LSTM-based DNN model gives better predictions than the LR, DT, SVM, and NN models, this must be supported by statistical analyses. Therefore, a non-parametric Mann-Whitney U test was employed to compare the prediction models at a significance level of 0.05 . The statistical results are presented in Table 5, where the mean difference and p-value are given to show which of the two prediction models is better. A p-value of less than 0.05 indicates a statistically significant difference. In terms of leakage, oil film thickness, and pocket pressure, there is a significant difference in favor of the proposed DNN model $(\mathrm{p} \leq 0.05)$. This shows

Table 3 Some descriptive statistics for $\mathbf{R}^{2}$ values

\begin{tabular}{|c|c|c|c|c|c|c|c|c|c|c|c|c|}
\hline & \multicolumn{4}{|c|}{ Leakage } & \multicolumn{4}{|c|}{ Oil film thickness } & \multicolumn{4}{|c|}{ Pocket pressure } \\
\hline & Mean & Max & Min & Std & Mean & Max & Min & Std & Mean & Max & Min & Std \\
\hline$L R$ & 0.9710 & 0.9735 & 0.9684 & 0.0012 & 0.9840 & 0.9861 & 0.9820 & 0.0010 & 0.9379 & 0.9468 & 0.9266 & 0.0047 \\
\hline DT & 0.9953 & 0.9986 & 0.9892 & 0.0024 & 0.9747 & 0.9881 & 0.9591 & 0.0079 & 0.9557 & 0.9658 & 0.9480 & 0.0045 \\
\hline SVM & 0.9926 & 0.9948 & 0.9900 & 0.0014 & 0.9880 & 0.9939 & 0.9785 & 0.0043 & 0.9555 & 0.9668 & 0.9398 & 0.0071 \\
\hline NN & 0.9952 & 0.9975 & 0.9928 & 0.0011 & 0.9977 & 0.9987 & 0.9955 & 0.0009 & 0.9584 & 0.9684 & 0.9412 & 0.0074 \\
\hline DNN & 0.9952 & 0.9981 & 0.9917 & 0.0015 & 0.9991 & 1.0000 & 0.9964 & 0.0010 & 0.9567 & 0.9657 & 0.9412 & 0.0061 \\
\hline
\end{tabular}

Bold values indicate the best score of the columns

Table 4 Some descriptive statistics for RMSE values

\begin{tabular}{|c|c|c|c|c|c|c|c|c|c|c|c|c|}
\hline & \multicolumn{4}{|c|}{ Leakage } & \multicolumn{4}{|c|}{ Oil film thickness } & \multicolumn{4}{|c|}{ Pocket pressure } \\
\hline & Mean & Max & Min & Std & Mean & Max & Min & Std & Mean & Max & Min & Std \\
\hline LR & 0.0085 & 0.0088 & 0.0082 & 0.0001 & 0.2753 & 0.2895 & 0.2596 & 0.0080 & 1.0135 & 1.1097 & 0.9289 & 0.0413 \\
\hline DT & 0.0033 & 0.0051 & 0.0019 & 0.0008 & 0.3328 & 0.4352 & 0.1855 & 0.0642 & 0.8593 & 0.9454 & 0.7490 & 0.0472 \\
\hline SVM & 0.0043 & 0.0050 & 0.0036 & 0.0004 & 0.2411 & 0.3261 & 0.1772 & 0.0383 & 0.8543 & 1.0101 & 0.7345 & 0.0727 \\
\hline NN & 0.0034 & 0.0046 & 0.0024 & 0.0006 & 0.0217 & 0.0987 & 0.0016 & 0.0269 & 0.8346 & 0.9712 & 0.7183 & 0.0605 \\
\hline DNN & 0.0028 & 0.0032 & 0.0021 & 0.0003 & 0.0037 & 0.0097 & 0.0015 & 0.0026 & 0.7696 & 0.7945 & 0.7130 & 0.0226 \\
\hline
\end{tabular}

Bold values indicate the best score of the columns 

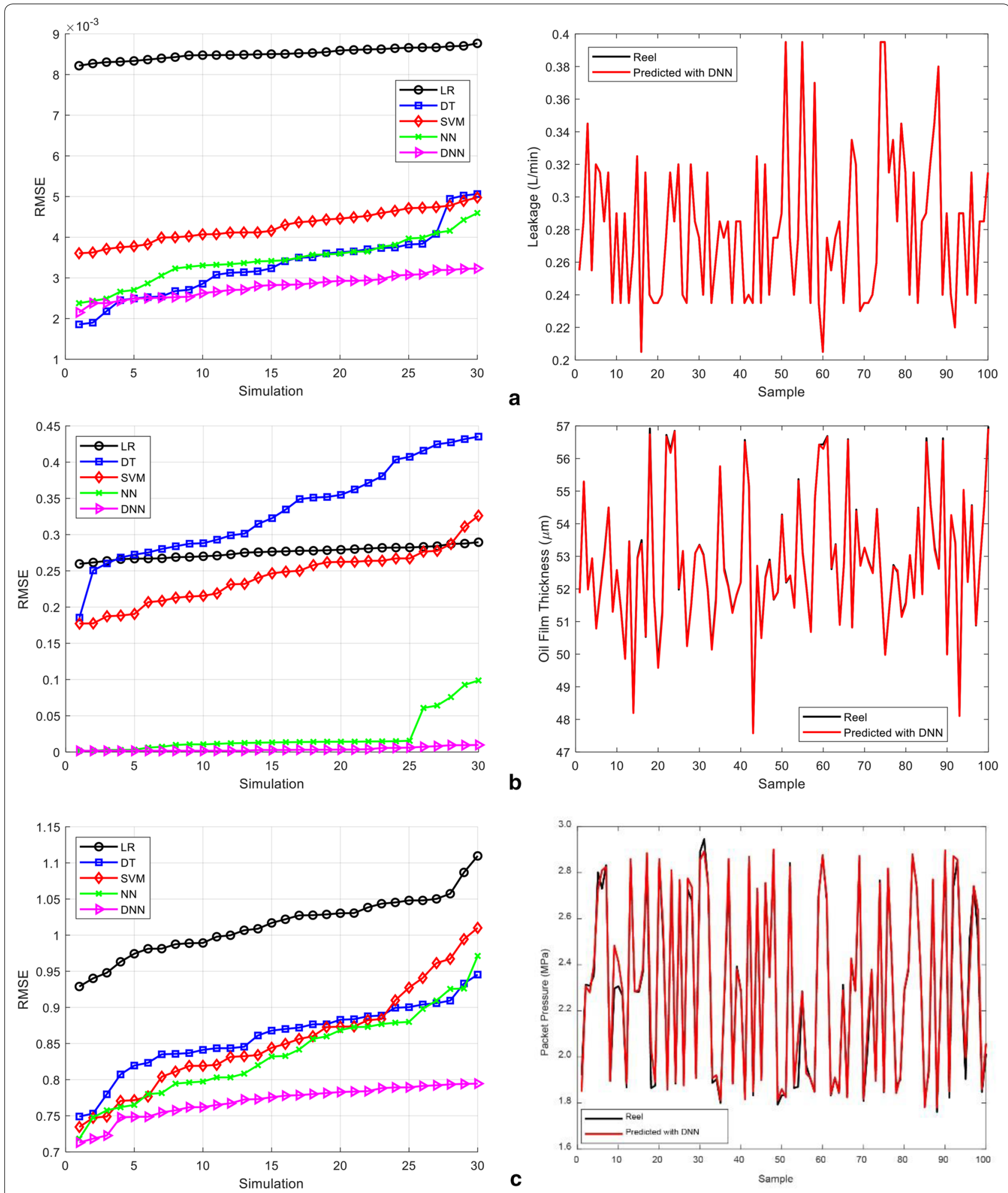

Figure 10 Comparison of MLMs for a leakage, $\mathbf{b}$ oil film thickness, and $\mathbf{c}$ pocket pressure 
Table 5 Mann-Whitney $\mathrm{U}$ test results are listed to compare the MLMs in terms of slipper parameters

\begin{tabular}{lllll}
\hline Comparison & Leakage & Oil film thick. & Pocket press. \\
\hline LR & Z & -6.653 & -6.653 & -6.653 \\
vs. & Mean Diff. & $5.730 \times 10^{-3}$ & $2.716 \times 10^{-1}$ & $2.439 \times 10^{-1}$ \\
DNN & p-value & 0.000 & 0.000 & 0.000 \\
& Sig. $(p<0.05)$ & $D N N$ & $D N N$ & $D N N$ \\
DT & Z & -2.957 & -6.653 & -5.751 \\
vs. & Mean Diff. & $5.230 \times 10^{-4}$ & $3.291 \times 10^{-1}$ & $8.965 \times 10^{-2}$ \\
DNN & $p$-value & 0.003 & 0.000 & 0.000 \\
& Sig. $(p<0.05)$ & $D N N$ & $D N N$ & $D N N$ \\
SVM & Z & -6.653 & -6.653 & -4.746 \\
vs. & Mean Diff. & $1.484 \times 10^{-3}$ & $2.374 \times 10^{-1}$ & $8.465 \times 10^{-2}$ \\
DNN & $p$-value & 0.000 & 0.000 & 0.000 \\
& Sig. $(p<0.05)$ & $D N N$ & $D N N$ & $D N N$ \\
NN & $Z$ & -4.613 & -5.131 & -4.539 \\
vs. & Mean Diff. & $6.511 \times 10^{-4}$ & $1.807 \times 10^{-2}$ & $6.496 \times 10^{-2}$ \\
DNN & $p$-value & 0.000 & 0.000 & 0.000 \\
& Sig. $(p<0.05)$ & $D N N$ & $D N N$ & $D N N$ \\
\hline
\end{tabular}

that the deep-LSTM model is considerably more stable than, and superior to, the other models.

\section{Conclusions}

Preventing metal-metal contact between the slipper and swash plate is important in terms of the general performance and lifespan of axial piston pumps. In this article, a slipper with circular dimples has been investigated in terms of the variation in leakage, oil film thickness, and pocket pressure under different working conditions. Dimples with a diameter and depth of $1 \mathrm{~mm}$ produced extra lifting force and elevated leakage and oil film thickness, thus reducing the pocket pressure value. As for circular dimples with a diameter of $1 \mathrm{~mm}$ and a depth of $2 \mathrm{~mm}$, they exhibited characteristics similar to those of flat slippers. Additionally, the experimental values were modeled using LSTM-based DNN, and the results were compared with those from LR, DT, SVM, and NN models. According to the results and statistical analyses, the proposed DNN approach yielded the best performance among all the prediction models studied. These results indicate that DNNs can be used by designers as a preliminary design tool. Different textures on the slipper provide additional lifting force at high pressures, and can behave like an oil reservoir under low pressures. In future studies, powerful machine learning algorithms can be used to examine the effect of sudden load variations, oil temperature, and working duration on slippers with different surface textures and geometries.
Acknowledgements

We would like to thank Editage [https://www.editage.com/] for editing and reviewing this manuscript for English language.

\section{Authors' Contributions}

ÖÖ was in charge of all experiments and also wrote the manuscript; $A C$ and $\mathrm{HB}$ were responsible for the machine learning methods and statistical analyses; CS provided necessary help with all parts of this article. All authors read and approved the final manuscript.

\section{Authors' Information \\ Özkan Özmen, born in 1985, is currently an assistant professor at Department of Industrial Design Engineering, Erciyes University, Turkey. His research interests include artificial intelligence in design optimization and computational fluid dynamics. \\ Cem Sinanoğlu, born in 1973, is currently a professor, Head of Department of Industrial Design Engineering and Vice Dean of Engineering Faculty, Erciyes University, Turkey. He has published numerous papers on lubrication, tribology, and the application of artificial intelligent. \\ Abdullah Caliskan, born in 1986, is with the Department of Biomedical Engi- neering, Iskenderun Technical University, Turkey, where he is an assistant profes- sor. His current research interests include signal processing, image processing, neural networks, deep learning, and the applications of these techniques. \\ Hasan Badem, born in 1986, is currently working at Department of Com- puter Engineering, Kahramanmaraş Sutcu Imam University, Turkey. His research interests include machine learning, especially deep learning, intelligent optimization, parallel and distributed computation, and the applications of these techniques.}

\section{Funding}

Supported by Erciyes University Scientific Research Projects Coordination Unit (Grant No. FDK-2016-6986).

\section{Availability of Data and Materials}

The data during the current study are available from the corresponding author on reasonable request.

\section{Competing Interests}

The authors declare that there are no conflicts of interest regarding the publication of this paper.

\section{Author Details}

${ }^{1}$ Tribology Laboratory, Industrial Design Engineering, Faculty of Engineering, Erciyes University, Kayseri 38039, Turkey. ${ }^{2}$ Biomedical Engineering, Faculty of Engineering and Natural Sciences, Iskenderun Technical University, Hatay, Turkey. ${ }^{3}$ Computer Engineering, Faculty of Engineering and Architecture, Kahramanmaraş Sutcu Imam University, Kahramanmaraş, Turkey.

Received: 17 January 2019 Revised: 4 February 2020 Accepted: 13 March 2020

Published online: 30 March 2020

\section{References}

[1] D Gropper, L Wang, T J Harvey. Hydrodynamic lubrication of textured surfaces: A review of modeling techniques and key findings. Tribology International, 2016, 94: 509-529.

[2] E Koc, C J Hooke. Considerations in the design of partially hydrostatic slipper bearings. Tribology International, 1997, 30(11): 815-823.

[3] E Koc, C J Hooke, KY Li. Slipper balance in axial piston pumps and motors Journal of Tribology, 1992, 114(4): 766-772.

[4] J M Bergada, J Watton, J M Haynes, et al. The hydrostatic/hydrodynamic behaviour of an axial piston pump slipper with multiple lands. Meccanica, 2010, 45(4): 585-602.

[5] J M Bergada, J M Haynes, J Watton. Leakage and groove pressure of an axial piston pump slipper with multiple lands. Tribology Transactions, 2008, 51 (4): 469-482.

[6] I Etsion, G Halperin, V Brizmer, et al. Experimental investigation of laser surface textured parallel thrust bearings. Tribology Letters, 2004, 17(2): 295-300. 
[7] SC Sharma, S K Yadav. A comparative study of full and partial textured hybrid orifice compensated circular thrust pad bearing system. Tribology International, 2016, 95: 170-180.

[8] S Ye, H Tang, Y Ren, et al. Study on the load-carrying capacity of surface textured slipper bearing of axial piston pump. Applied Mathematical Modelling, 2020, 77: 554-584.

[9] M A Karkoub, O E Gad, M G Rabie. Predicting axial piston pump performance using neural networks. Mechanism and Machine Theory, 1999, 34(8): 1211-1226.

[10] M Karkoub, A Elkamel. Modelling pressure distribution in a rectangular gas bearing using neural networks. Tribology International, 1997, 30(2): 139-150.

[11] F Canbulut, C Sinanoğlu, Ş Yıldırım, et al. Design of neural network model for analyzing hydrostatic circular recessed bearings with axial piston pump slipper. Industrial Lubrication and Tribology, 2004, 56(5): 288-299.

[12] F Canbulut, C Sinanoğlu, Ş Yildirim. Neural network analysis of leakage oil quantity in the design of partially hydrostatic slipper bearings. Industrial Lubrication and Tribology, 2004, 56(4): 231-243.

[13] F Canbulut, E Koç, C Sinanoğlu. Design of artificial neural networks for slipper analysis of axial piston pumps. Industrial Lubrication and Tribology, 2009, 61(2): 67-77

[14] F Canbulut, \$̧ Yildirim, C Sinanoğlu. Design of an artificial neural network for analysis of frictional power loss of hydrostatic slipper bearings. Tribology Letters, 2004, 17(4): 887-899.

[15] Ö Özmen, C Sinanoğlu, T Batbat, et al. Prediction of slipper pressure distribution and leakage behaviour in axial piston pumps using ANN and MGGP. Mathematical Problems in Engineering, 2019, https://doi. org/10.1155/2019/7317520.

[16] F Canbulut, C Sinanoğlu, M Erkilet, et al. Neural predictor to analyse the efficiency of positive displacement pumps. Journal of the Balkan Tribological Association, 2012, 18(4): 530-539.

[17] Y LeCun, Y Bengio, G Hinton. Deep learning. Nature, 2015, 521(7553): 436-444.
[18] Q Chao, J Zhang, B Xu, et al. Test rigs and experimental studies of the slipper bearing in axial piston pumps: A review. Measurement, 2019, 132: 135-149.

[19] S Kumar, J M Bergada, J Watton. Axial piston pump grooved slipper analysis by CFD simulation of three-dimensional NVS equation in cylindrical coordinates. Computers \& Fluids, 2009, 38(3): 648-663.

[20] Caliskan, M E Yuksel, H Badem, et al. A deep neural network classifier for decoding human brain activity based on magnetoencephalography. Elektronika ir Elektrotechnika, 2017, 23(2): 63-67.

[21] H Badem, A Caliskan, A Basturk, et al. Classification and diagnosis of the Parkinson disease by stacked autoencoder. ELECO 2016 - IEEE National Conference on Electrical, Electronics and Biomedical Engineering, Bursa, Turkey, 2016: 499-502.

[22] A Caliskan, M E Yuksel, H Badem, et al. Performance improvement of deep neural network classifiers by a simple training strategy. Engineering Applications of Artificial Intelligence, 2018, 67: 14-23.

[23] H Badem, A Basturk, A Caliskan, et al. A new efficient training strategy for deep neural networks by hybridization of artificial bee colony and limited-memory BFGS optimization algorithms. Neurocomputing, 2017, 266: 506-526.

[24] R Jozefowicz, W Zaremba, I Sutskever. An empirical exploration of recurrent network architectures. ICML 2015 - International Conference on Machine Learning, Lille, France, 2015: 2342-2350.

[25] S Hochreiter, J Schmidhuber. Long short-term memory. Neural Computation, 1997, 9(8): 1735-1780.

[26] H Sak, A Senior, F Beaufays. Long short-term memory recurrent neural network architectures for large scale acoustic modeling. INTERSPEECH 2014, In Fifteenth Annual Conference of the International Speech Communication Association, Singapore, 2014: 338-342

[27] F Canbulut, C Sinanoğlu, E Koç. Experimental analysis of frictional power loss of hydrostatic slipper bearings. Industrial Lubrication and Tribology, 2009, 61(3): 123-131.

\section{Submit your manuscript to a SpringerOpen ${ }^{\circ}$ journal and benefit from:}

- Convenient online submission

- Rigorous peer review

- Open access: articles freely available online

- High visibility within the field

- Retaining the copyright to your article

Submit your next manuscript at springeropen.com 